\title{
Spherical Complexes and Nonprojective Toric Varieties
}

\author{
Günter Ewald \\ Institut für Mathematik, Ruhr-Universität Bochum, Postfach 102148, 4630 Bochum 1, \\ Federal Republic of Germany
}

\begin{abstract}
A combinatorial criterion for a toric variety to be projective is given which uses Gale-transforms. Furthermore, classes of nonprojective toric varieties are constructed.
\end{abstract}

\section{Introduction}

Let $\sigma:=\mathbb{R}_{+} a_{1}+\cdots+\mathbb{R}_{+} a_{k}$ be a cone in $\mathbb{R}^{d}$, where $a_{1}, \ldots, a_{k}$ are primitive lattice points $\in \mathbb{Z}^{d} \backslash\{0\}$, and let $\sigma$ have only 0 as an apex. If $S$ is the unit sphere in $\mathbb{R}^{d}$, the intersection $\sigma_{0}:=\sigma \cap S$ is a spherical cell. Suppose $\Sigma_{0}$ is a spherical cell complex consisting of such cells. The corresponding cones form a system $\Sigma$ called a fan. We can assume that every point $\left(\mathbb{R}_{+} a_{j}\right) \cap S$ is a vertex of $\sigma_{0}$ for any $\sigma_{0} \in \Sigma_{0}$. We may also consider $\Sigma$ to be a cell complex, the vertices being one-dimensional cones $\mathbb{R}_{+} a_{j}$.

If $\operatorname{dim} \sigma:=\operatorname{dim}\left(\right.$ aff $\sigma$ ) (affine hull) equals $k$ we call $\sigma$ and $\sigma_{0}$ simplicial. We say $\Sigma$ or $\Sigma_{0}$ is simplicial if every $\sigma \in \Sigma$ or $\sigma_{0} \in \Sigma_{0}$ is simplicial, respectively. In the case of a simplicial fan we also look at $\Sigma$ as being generated by projecting the simplexes $\sigma^{\prime}:=\operatorname{con} v\left\{a_{1}, \ldots, a_{k}\right\}$, that is, $\sigma=\mathbb{R}_{+} \boldsymbol{\sigma}^{\prime}$ for all $\sigma \in \Sigma$. The simplicial complex $B_{\mathrm{st}}(Q)$ of all $\boldsymbol{\sigma}^{\prime}$ thus defined bounds a star-shaped polyhedron $Q$ with 0 in its kernel, provided $\Sigma$ covers the whole space $\mathbb{R}^{d}$. Let $\check{\sigma}:=\{x \mid\langle x, y\rangle \geq 0$ for all $y \in \sigma\}$ be the dual cone of $\sigma\left(\langle\cdot, \cdot\rangle=\right.$ inner product), and let $R_{\sigma}$ be the ring of all Laurent-polynomials $\sum a_{j} \mathbf{z}^{j}, a_{j} \in C$ (or any algebraically closed field), $\mathbf{z}^{j}:=\mathbf{z}_{1}^{j_{1}}$ $\cdots \mathbf{z}_{d}^{j_{d}}, j:=\left(j_{1}, \ldots, j_{d}\right) \in \sigma^{\circ} \cap \mathbf{Z}^{d}$, only finitely many $a_{j}$ being $\neq 0$. Spec $R_{\sigma}$ (the set of prime ideals of $R_{a}$ ) is an affine variety. For any two $\sigma_{1}, \sigma_{2} \in \Sigma$ we glue together $\operatorname{Spec} R_{\sigma_{1}}$ and $\operatorname{Spec} R_{\sigma_{2}}$ by the inclusion maps

$$
R_{\sigma_{1} \cap \sigma_{2}} \leftarrow R_{\sigma_{1}}, \quad R_{\sigma_{1} \cap \sigma_{2}} \leftarrow R_{\sigma_{2}} .
$$

If this is done for all $\sigma_{1}, \sigma_{2} \in \Sigma$ we obtain a variety $X_{\Sigma}$ called toric variety (see 
Kempf, Knudson, Mumford, and Saint-Donat [6], Oda [10], Danilov [2], and Teissier [14]; also [3]).

Any fan can easily be extended to a fan that covers all of $\mathbb{R}^{d}$. For $X_{\Sigma}$ this means a compactification (completion). We assume in this article $\Sigma$ to cover $\mathbb{R}^{d}$ and hence $\Sigma_{0}$ to have the sphere as its point set.

Our main goal is to extend some of the work of Oda and Miyake [10,11] from three to higher dimensions. In particular, we study questions of projectiveness of $X_{\Sigma}$ and construct classes of nonprojective toric varieties in all dimensions. We make use of the technique of the so-called Gale-transforms which proved to be very helpful in combinatorial convexity theory.

In the "dictionary" that relates properties of $\Sigma$ to properties of $X_{\Sigma}$ we focus on three "words":

1. For $d=2, \Sigma$ can also be obtained by projecting the faces of a convex polyhedron $P$ (see Fig. 1). For $d>2$, this is, in general, not true. If it is true, we say $\Sigma$ is strongly polytopal. $X_{\Sigma}$ is called projective if it is globally the set of zeros of finitely many homogeneous polynomials in $d+1$ variables. The following equivalence is true (see for example, [2], page 118):

$\Sigma$ strongly polytopal $\Leftrightarrow X_{\Sigma}$ projective.

2. If $\sigma$ is simplicial and if $\operatorname{dim} \sigma=d$, that is, $\sigma \in \Sigma^{(d)}$, we assign to the generating vectors $a_{1}, \ldots, a_{d}$ the determinant $\operatorname{det} \sigma:=\operatorname{det}\left[a_{1}, \ldots, a_{d}\right]$. It can be shown ([10], page 12)

$$
\operatorname{det} \sigma= \pm 1 \text { for all } \sigma \in \Sigma^{(d)} \Leftrightarrow X_{\Sigma} \text { is nonsingular. }
$$

3. If in a cell complex $\mathscr{C}$ we choose a relative interior point $p$ of a cell $C$ ( $p \in \operatorname{relint} C$ ), and if the star of $C$ is replaced by the join of $p$ to the boundary of this star, we say, a stellar subdivision $s(p, \mathscr{C})$ has been achieved (Fig. 2). We call a stellar subdivision $S\left(\mathbb{R}_{+} a, \Sigma\right)$ regular if $a=a_{1}+\cdots+a_{k}$ for $a_{1}, \ldots, a_{k}$ generating a cone of $\Sigma$. (The term "barycentric" used by Oda and Miyake is somewhat misleading.) There is a correspondence (see [10]):

$$
\begin{aligned}
\text { (regular) stellar subdivision of } \Sigma \rightarrow & \text { blow-up of } X_{\Sigma} \text { (along a } \\
& \text { nonsingular center) }
\end{aligned}
$$

The inverse operation of a blow-up is called a blow-down (or o-process).

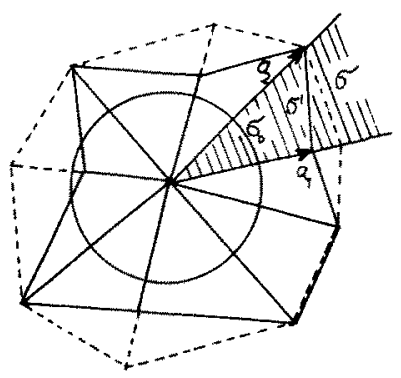

Fig. 1 

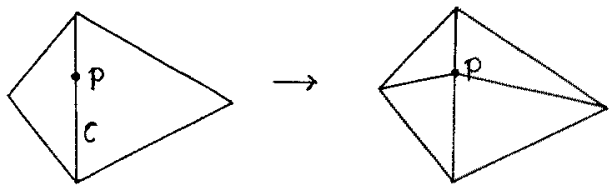

Fig. 2

\section{Gale-Transforms and Facet-Splitting}

Let $V:==\left\{a_{1}, \ldots, a_{v}\right\}$ be a finite set of points (vectors) in $\mathbb{R}^{d}$, and let $\left(\alpha_{1}, \ldots, \alpha_{v}\right)$ be an affine dependence of $V$, that is,

$$
\alpha_{1} a_{1}+\cdots+\alpha_{v} a_{v}=0, \quad \alpha_{1}+\cdots+\alpha_{v}=0 .
$$

We choose a basis of the $(v-d-1)$-dimensional space of all affine dependences and write them as rows of a matrix

$$
\left(\begin{array}{ccc}
\alpha_{11} & \cdots & a_{1, v} \\
\vdots & & \vdots \\
\alpha_{v-d-1,1} & \cdots & \alpha_{v-d-1, v}
\end{array}\right)=:\left(\bar{a}_{1}, \ldots, \bar{a}_{v}\right) .
$$

The set of columns $\bar{V}:=\left\{\bar{a}_{1}, \ldots, \bar{a}_{v}\right\}$ is called a Gale-transform of $V$ (see, for example, Grünbaum [5] or McMullen and Shephard [9], Ewald and Voß [4], and, for a coordinate-free introduction, McMullen [8]).

Example. Consider in $\mathbb{R}^{3}$ the triangular prism with vertices $a_{1}=(1,0,0), a_{2}=$ $(0,1,0), a_{3}=(0,0,1), a_{4}=(0,-1,-1), a_{5}=(-1,0,-1)$, and $a_{6}=(-1,-1,0)$. Let the rectangular faces be split as indicated in Fig. 3. Figure 4 presents a Gale-transform of $V=\left\{a_{1}, \ldots, a_{6}\right\}$. If $a_{i_{1}}, \ldots, a_{i_{k}}$ generate a cell ("face") $\sigma$ of $\Sigma$ we call $\bar{V} \backslash\left\{\bar{a}_{i_{1}}, \ldots, \bar{a}_{i_{k}}\right\}$ the coface $\bar{\sigma}$ of $\sigma$. We make use of a basic fact [13]:

Theorem. $\quad \Sigma$ is strongly polytopal if and only if $\bigcap_{\sigma \in \Sigma}$ relint $\bar{\sigma} \neq \varnothing$.

If, in particular, $0 \in \bigcap_{\sigma \in \Sigma}$ rel int $\bar{\sigma}$, then $a_{1}, \ldots, a_{v}$ represent the vertices of a convex polytope.

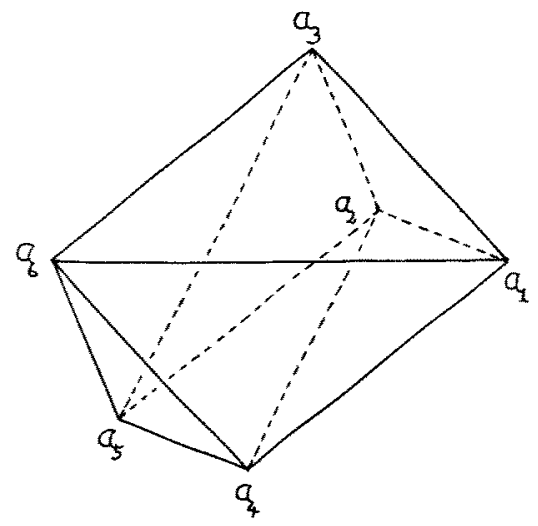

Fig. 3 


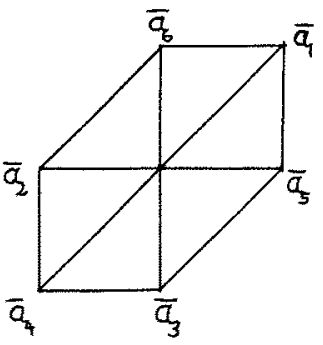

Fig. 4

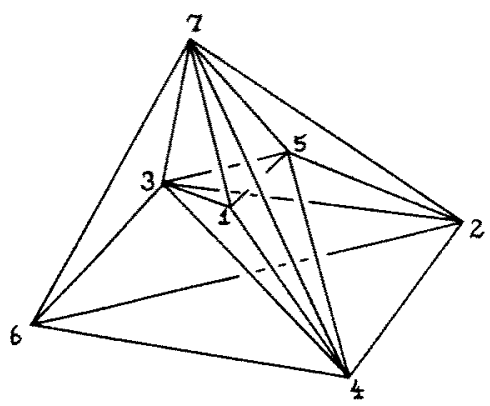

Fig. 5

In the above example, the prism without face-splitting has a face-structure that satisfies the latter condition. If the splittings are carried out, however, among the cofaces there are $\bar{a}_{1} \bar{a}_{4} \bar{a}_{6}, \bar{a}_{2} \bar{a}_{5} \bar{a}_{4}, \bar{a}_{3} \bar{a}_{6} \bar{a}_{5}$ which have no relative interior point in common. So we obtain a nonstrongly polytopal fan $\Sigma$.

All determinants of $\Sigma$ except $\operatorname{det}\left[a_{4}, a_{5}, a_{6}\right]$ are \pm 1 . Applying the regular stellar subdivision, $S\left(\mathbb{R}_{+} a, \Sigma\right)$ where $a=a_{4}+a_{5}+a_{6}$ provides a nonsingular, nonprojective variety $X_{S\left(\mathbf{R}_{+} a, \Sigma\right) \Sigma}$.

An analogous construction for $d=4$ can be obtained as follows. Consider the subdivision of a three-simplex as indicated in Fig. 5. It consists of double-simplexes $\Delta_{1}:=12457, \Delta_{2}:=23567, \Delta_{3}:=31647$, and four simplexes 1435, 2346, 1235, 1357. A Gale-transform $\overline{1}, \ldots, \overline{7}$ of $1, \ldots, 7$ is shown in Fig. 6. This decomposition of the simplex can be looked at as the Schlegel-diagram of a four-polytope $P$, that is, a central projection of $P$ into one of its facets. A direct construction of $P$ can be obtained by finding a Gale-transform of the points in Fig. 6 and taking their convex hull. It is known that Fig. 6 represents aoain a

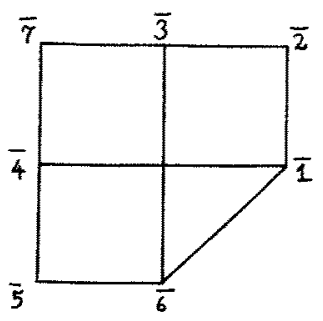

Fig. 6 


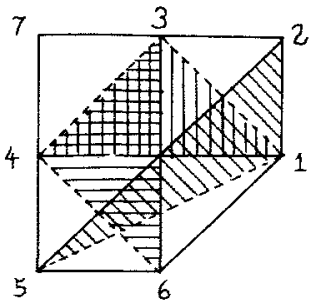

Fig. 7

Gale-transform of $P$. The double-simplexes $\Delta_{1}, \Delta_{2}, \Delta_{3}$ can be looked at as analogues of the rectangular faces of the prism in Fig. 3 which are two-dimensional double-simplexes.

Now we may split each of the facets $\Delta_{1}, \Delta_{2}, \Delta_{3}$ by a one-dimensional or a two-dimensional diagonal into three or two simplexes. There are eight typical combinations of such facet-splittings, two of which turn out to provide nonstrongly polytopal fans:

I. Split $\Delta_{1}$ at $12, \Delta_{2}$ at 56 , and $\Delta_{3}$ and 347 ;

II. split $\Delta_{1}$ at $457, \Delta_{2}$ at 237 , and $\Delta_{3}$ at 16 .

Figures 7 and 8 provide for cases I and II, respectively, three cofaces that have no relative interior point in common.

For any $d \geq 3$ we obtain the following statement. By facet-splitting we mean generally the straight subdivision of the facets of a convex polytope into convex polytopes whose vertices are all vertices of the original polytope.

Theorem 1. Let $P$ be a convex $d$-polytope, $d \geq 3,0 \in$ int $P$, with $v$ rational vertices, and let $P$ have at least $v-d$ facets which are simplicial but not simplexes. Then by appropriate facet-splittings we obtain at least one complex $B(P)$ on the boundary of $P$ such that $\Sigma=\Sigma(B(P))$ is not strongly polytopal.

Proof. A Gale-transform of the vertex set vert $P$ of $P$ spans a space of dimension $v-d-1$. Let $\Delta_{1}^{\prime}, \ldots, \Delta_{v-d}^{\prime}$ be simplicial facets that are not simplexes. If $\Delta_{j}^{\prime}$ has more than $d+1$ vertices, we apply facet-splittings until we obtain a piece $\Delta_{j}$ of $\Delta_{j}^{\prime}$ that has precisely $d+1$ vertices. So let $\Delta_{1}, \ldots, \Delta_{v-d}$ be $(d-1)$-cells of a cell-complex $B_{0}(P)$ realized on the boundary of $P$.

To each $\Delta_{j}$ let $\bar{\Delta}_{j}$ be a coface which is $(v-d-2)$-dimensional and hence spans a hyperplane $H_{j}$ in $\mathbb{R}^{v-d-1}$. Now $\Delta_{j}$ can be split into simplexes using a Radon partition of vert $\Delta_{j}$ into subsets $D_{j}, D_{j}^{\prime}$ such that $D_{j} \cup D_{j}^{\prime}=\operatorname{vert} \Delta_{j}$,

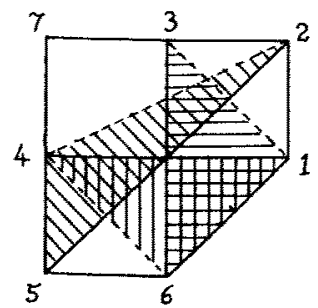


$D_{j} \cap D_{j}^{\prime}=\varnothing,\left(\right.$ conv $\left.D_{j}\right) \cap\left(\operatorname{conv} D_{j}^{\prime}\right) \neq \varnothing$. We obtain $(v-d-1)$-dimensional cofaces conv $\left(\bar{\Delta}_{j} \cup\left\{\bar{a}_{i}\right\}\right), a_{i} \in$ vert $\Delta_{j}$, which can lie on either side of $H_{j}$ depending on whether $a_{i} \in D_{j}$ or $a_{i} \in D_{j}^{\prime}$. Since $\Delta_{1}^{\prime}, \ldots, \Delta_{v-d}^{\prime}$ are simplicial, all splittings of the $\Delta_{j}$ are independent. Hence they can be chosen in such a way that

$$
\bigcap_{j=1}^{\prime-d} \operatorname{relint} \operatorname{conv}\left(\bar{\Delta}_{j} \cup\left\{\bar{a}_{i,}\right\}\right)=\varnothing .
$$

By Shephard's theorem, this proves our assertion.

Remark 1. Polytopes $P$ as assumed in Theorem 1 do exist for any $d \geq 3$. Let, for example, $C$ be a $d$-dimensional cube with 0 as its center, and consider in any one-dimensional face $p q$ of $C$ the supporting hyperplane $H$ such that $H \cap C=p q$ and such that $H$ is perpendicular to the plane spanned by $0, p$, and $q$. Then the half-spaces bounded by such $H$ and containing 0 intersect in a polytope $P$ that has $v=2^{d}+2 d$ vertices and $d \cdot 2^{d-1}$ simplicial facets that are not simplexes. Since $v-d=2^{d}+d<d \cdot 2^{d-1}$ for $d \geq 3$ there are sufficiently many such faces available. Further examples for $d=4$ can be found in Altshuler and Steinberg [1].

Remark 2. In many cases there will be more than one nonstrongly polytopal fan that can be constructed from $P$. If, for example, $d$ is even and $v-d$ is odd, then the two possible facet-splittings of $\Delta_{j}$ are nonisomorphic. Replacing $\operatorname{conv}\left(\bar{\Delta}_{j} \cup\right.$ $\left.\left\{a_{i}\right\}\right)$ by conv $\left(\bar{\Delta}_{j} \cup\left\{a_{k_{f}}\right\}\right)$ where $a_{i,}, a_{k_{j}}$ are in different sets $D_{j}, D_{j}^{\prime}, j=1, \ldots$, $v-d$, provides a fan that is nonisomorphic to the first one. This example generalizes cases I and II in the above four-dimensional example.

Remark 3. If the hyperplanes $H_{j}$ are linearly dependent, then, in general, less than $v-d$ facet splittings will do to obtain nonstrongly polytopal fans. The same is true in many cases where the $\Delta_{j}$ have more than $d+1$ vertices.

\section{Canonical Extensions}

We present now a further method of constructing nonprojective toric varieties from given ones. If the variety $X_{\Sigma}$ we start with has no singularities the same is true for the new ones. Also the possibility of turning the variety into a projective space by blow-ups and -downs is preserved.

Let $\Sigma$ be a simplicial fan in $\mathbb{R}^{d}$, and let $V:=\left\{a_{1}, \ldots, a_{v}\right\}$ be the set of its generating primitive lattice vectors. We embed $\mathbb{R}^{d}$ into $\mathbb{R}^{d+1}$, replace a vertex $\left(a_{j}, 0\right)$, say $\left(a_{1}, 0\right)$, by $\left(a_{1}, 1\right)$, and join $\left(a_{1}, 1\right)$ to the complement of the star of $\left(a_{1}, 0\right)$ in the complex $B_{\text {st }}(Q)$ on the boundary of the star-shaped polytope such that $\Sigma$ projects the faces of $B_{\text {st }}(Q)$ (see Section 1 ). Then we join $(0,-1)$ to the boundary of the complex thus constructed. We obtain a complex $\tilde{B}_{\text {st }}(\tilde{Q})$ which bounds a star-shaped polytope $\tilde{Q}$ in $\mathbb{R}^{d+1}$. We call $\tilde{B}_{\text {st }}(\tilde{Q})$ or its associated fan $\tilde{\Sigma}$ a canonical extension of $B_{\text {st }}(Q)$ or $\Sigma$, respectively. Also $X_{\tilde{\Sigma}}$ is then called a canonical extension of $X_{\Sigma}$ (Fig. 9). (According to Provan and Billera [12] $\tilde{B}_{\text {st }}(\tilde{Q})$ is the simplicial wedge of $B_{\mathrm{st}}(Q)$ on $a_{1}$; according to Klee and Kleinschmidt [7] the dual wedge.) 


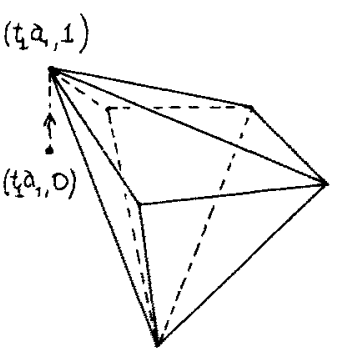

$(0,-t)$
Fig. 9

$\tilde{B}_{\text {st }}(\tilde{Q})$ can also be obtained by doubling $\bar{a}_{1}$ in a Gale-transform $\bar{V}$ of $V: \bar{a}_{1}=\bar{a}_{v+1}$. The additional affine dependence $\bar{a}_{1}-\bar{a}_{v+1}=0$ provides us the new vertices $\left(a_{1}, 1\right),\left(a_{2}, 0\right), \ldots,\left(a_{v}, 0\right),(0,-1)$ in the extended original space. This interpretation of the canonical extension should be kept in mind but is not necessary for what follows.

Theorem 2. Let $X_{\tilde{\Sigma}}$ be a canonical extension of $X_{\Sigma}$.

(1) If $X_{\Sigma}$ has dimension $d, X_{\Sigma}$ has dimension $d+1$.

(2) If $X_{\Sigma}$ is projective, so is $X_{\tilde{\Sigma}}$.

(3) If $X_{\Sigma}$ is nonprojective, so is $X_{\tilde{\Sigma}}$.

(4) If $X_{\Sigma}$ is nonsingular and can, by blow-ups and -downs, be transformed into a projective space, the same is true for $X_{\tilde{\Sigma}}$.

Proof. (1) True, by definition.

(2) Let $t_{1} a_{1}, \ldots, t_{v} a_{v}, t_{j}>0, j=1, \ldots, v$, be vertices of a convex polytope. Then $\left(t, a_{1}, 0\right)$ is outside $P_{0}:=\operatorname{conv}\left\{\left(t_{1} a_{1}, 1\right),\left(t_{2} a_{2}, 0\right), \ldots,\left(t_{v} a_{v}, 0\right)\right\}$. Hence, if $t>0$ is sufficiently large, the line segment joining $\left(t_{1} a_{1}, 1\right)$ and $(0,-t)$ is also outside $P_{0}$. Therefore, $\tilde{\Sigma}$ is also strongly polytopal, the realizing polytope being $\operatorname{conv}\left(P_{0}\right.$ $\cup\{0,-t\})$.

(3) Suppose $\tilde{\Sigma}$ were strongly polytopal, being realized by a polytope $\tilde{P}$. Then $P:=\tilde{P} \cap\left\{X_{d+1}=0\right\}$ is a realization for $\Sigma$, a contradiction.

(4) The determinants of $d+1$ rows associated with facets of $P_{0}$ evidently reduce, up to a factor \pm 1 , to determinants of $d$ rows associated with the facets of $B_{\mathrm{st}}(Q)$, hence are \pm 1 .

We apply first a stellar subdivision $S\left(\mathbb{R}_{+} p, \tilde{\Sigma}\right)$ where $p=\left(a_{1}, 0\right)+(0,-1)$. The complex $\mathscr{C}^{\prime}:=\left[B_{\text {st }}(Q) \backslash \operatorname{star}\left(a_{1}, B(Q)\right)\right] \cup\left[p \cdot\right.$ link $\left.\operatorname{star}\left(a_{1}, B(Q)\right)\right](p \cdot \mathscr{C}:=$ $\{\operatorname{conv}(\{p\} \cup \sigma) \mid \sigma \in \mathscr{C}\}$ the join of $p$ and $\mathscr{C})$ is isomorphic to $B(Q)$. Hence regular stellar subdivisions and inverses applied successively to $B_{\text {st }}(Q)$ correspond to analogous operations for $\mathscr{C}^{\prime}$ and can naturally be extended to operations for $\tilde{B}_{\mathrm{st}}(\tilde{Q})$. If $B_{\mathrm{st}}(Q)$ is thus transformed into a $d$-simplex, $\tilde{B}_{\mathrm{st}}(\tilde{Q})$ is being transformed into a double-simplex which, in turn, is readily transformed into a simplex. (Compare Provan and Billera [12] and Klee and Kleinschmidt [7].)

Theorem 2 provides a construction method for nonprojective toric varieties in all dimensions $d>3$. In particular, we have from the examples presented in 


\section{Section 2:}

Theorem 3. (1) For any $d \geq 3$ there exist nonprojective toric varieties with $v=d+3$ exceptional divisors.

(2) For any $d \geq 3$ there exist nonsingular, nonprojective toric varieties having $v=d+4$ exceptional divisors.

Remark. If $X_{\Sigma}$ can be blown down, this only carries over to $X_{\tilde{\Sigma}}$ if $\mathbb{R}_{+} a_{1} \neq \mathbb{R}_{+} p$ in $S\left(\mathbb{R}_{+} p, \Sigma\right)$.

\section{References}

1. A. Altshuler and L. Steinberg, Enumeration of the quasisimplicial 3-spheres and 4-polytopes with eight vertices, manuscript.

2. V. I. Danilov, The geometry of toric varieties. Uspekhé Nauk 33 (1978) 85-134, Russian Math. Surveys 33 (1978), 97-154.

3. G. Ewald, Torische Varietäten und konvexe Polytope. To appear in Studia Scientiarum Mathematicarum Hungarica, Budapest.

4. G. Ewald and K. Voß, Konvexe Polytope mit Symmetriegruppe. Comm. Math. Helvet. 48 (1973), 137-150.

5. B. Grünbaum, Convex Polytopes, John Wiley, 1967.

6. G. Kempf, F. Knudson, D. Mumford, and B. Saint-Donat, Toroidal Embeddings I. Lecture Notes in Mathematics, Springer-Verlag, Berlin, 1973.

7. V. Klee and P. Kleinschmidt, The $d$-step conjecture and its relatives, manuscript.

8. P. McMullen, Transforms, diagrams and representations, in Contributions to Geometry (Proc. Geom. Symp. Siegen 1978), 92-130.

9. P. McMullen and G. C. Shephard, Convex Polytopes and the Upper Bound Conjecture, Cambridge Univ. Press, 1971.

10. T. Oda, Torus Embeddings and Applications, Tata Institute, Bombay, 1978.

11. T. Oda and K. Miyake, Almost homogeneous algebraic varieties under algebraic torus action. Manifolds-Tokyo 1973 (Hattori, ed.), Univ. of Tokyo Press 1975, 373-381.

12. J. S. Provan and G. J. Billera, Decompositions of simplicial complexes related to diameters of convex polyhedra. Math. Operations Research, 5 (1980), 576-594.

13. G. C. Shephard, Spherical complexes and radial projections of polytopes, Israel J. of Math. 9 (1971), 257-262.

14. B. Teissier, Variétés toriques et polytopes. Seminaire Bourbaki, 33e. année (1980/81), Nr. 565. 1-14.

Received July 3, 1985. 